\title{
Early hospital discharge of newborn infants was not associated with feeding related hospital readmission during the first 28 days of life
}

Edmonson MB, Stoddard JJ, Owens LM. Hospital readmission with feeding-related problems after early postpartum discharge of normal newborns. JAMA 1997 Jul 23/30;278:299-303.

\section{Objective}

To determine whether an association exists between early postpartum discharge of newborn infants and feeding related hospital readmission during the first 28 days of life.

\section{Design}

Population based, nested case control study, with record linkage of computerised birth certificate data, statewide hospital discharge data, and data on neonatal feeding from the Wisconsin Newborn Screening Program.

\section{Setting}

State of Wisconsin, USA.

\section{Participants}

210 case patients (newborn infants readmitted to hospital with feeding related problems at age 4-28 d) and 630 unmatched control infants (no feeding related admissions) were identified from a cohort of 120290 singleton newborn infants who were delivered vaginally between 1 January 1991 and 31 October 1994 , with birth weights $\geqslant 2500 \mathrm{~g}$, continuous hospital stays until discharge, receipt of normal newborn care, and postpartum discharge records that could be linked to birth certificate data. Exclusion criteria were congenital anomalies, abnormal postpartum conditions, hospital transfer of mother before delivery, complications during pregnancy, labour or delivery, or postpartum maternal sterilisation.

\section{Assessment of risk factors}

Early discharge was defined as discharge from the birth hospital on the day of birth (day 1) or the next day (day 2). Conventional discharge was defined as discharge on day 3 .

\section{Main outcome measure}

Feeding related hospital readmission at age 4-28 days, defined as direct admission of a newborn infant (who had been previously discharged from the birth hospital to home) with $\geqslant 1$ International Classification of Diseases, Ninth Revision, Clinical Modification (ICD-9-CM) discharge diagnosis code indicating a feeding problem, dehydration, or inadequate weight gain, and no ICD-9-CM code indicating a specific aetiology or disease process to which the above problems could be attributed.

\section{Main results}

255 newborn infants $(31 \%)$ were discharged early from hospital. Of those infants readmitted for feeding related problems (case patients), 69 (33\%) had been discharged early (day 1 or 2) compared with $186(30 \%)$ infants in the control group. Feeding related readmission was not associated with early discharge (odds ratio [OR] $1.05,95 \%$ CI 0.71 to 1.53 , adjusted for sex, birth weight, preterm delivery, type of feeding, medical assistance, health maintenance organisation, maternal age, race/ethnicity, education, marital status, parity, first trimester care, place of residence, and year of delivery). Feeding related hospital readmission was independently associated with breast feeding (OR 2.6), preterm delivery (OR 2.3), primiparity (OR 1.8), low maternal education (OR 1.7), and Medicaid insurance (OR 1.7) (all $\mathrm{p}<0.05)$.

\section{Conclusion}

Early discharge of newborn infants from the birth hospital on the day of birth, or the day after birth, was not associated with increased feeding related hospital readmissions during the first 28 days.

Source of funding: not stated.

For article reprint:Dr M B Edmonson, Department of Pediatrics, H6/440 Clinical Science Center, 600 Highland Avenue, Madison, WI 53792-4116, USA. Fax +1 6082630440

\section{Commentary}

Postpartum hospital stays have decreased dramatically over the past few decades to the present 12-48 hours for vaginal births and 4 days for caesarean births. Although initiated in response to consumer demands to "demedicalise" childbirth, this trend has continued and has intensified in response to pressures of cost containment.

Numerous studies have explored the safety of early postpartum discharge. Critical reviews of the literature have failed to reveal the optimum length of postpartum stay because of serious methodological flaws in the primary research including variable definitions of early discharge; small sample sizes with limited power; inadequate study designs lacking randomisation or appropriate control or comparison groups; limited generalisability because of selection bias, different inclusion criteria, and a variety of interventions before and after discharge; and diverse outcomes. ${ }^{12}$

The studies by Liu et al and Edmonson et al explore the question of safety, while addressing the limitations of previous studies. Both studies are well designed and use similar methods, data sources, and time periods. They are population based, with case control designs and large sample sizes, enabling broad generalisability and increased power to draw meaningful conclusions. There initially appears to be a discrepancy in their results, but on further review this is because Liu et al looked at readmissions in general, whereas Edmonson et al looked only at feeding related readmissions, thus having a smaller sample size and lacking the statistical power to detect differences of the same magnitude. The 1 limitation associated with both studies is that no information is provided on predischarge or postdischarge practices, both of which can contribute to the safety of early discharge.

The results of these studies are important for postpartum nurses working in hospital and in the community. There are implications for clinical practice, education, research advocacy, and policy development.

Although most newborn babies remained healthy, there was an increased risk of readmission with early discharge in certain subgroups. This finding reinforces the need for nurses to carefully assess mothers (continued on page 42) 


\title{
Early hospital discharge of newborn infants was associated with increased risk of hospital readmission during the first 28 days of life
}

\author{
Liu LL, Clemens CJ, Shay DK, et al. The safety of newborn early discharge. The Washington state experience. JAMA 1997 Jul
} 23/30;278:293-8.

\section{Objective}

To determine whether early discharge of newborn infants from hospital is associated with increased risk of readmission during the first 28 days of life.

\section{Design}

Population based, case control study, with linkage of records from the Washington State Birth Events Record Database and the Comprehensive Hospital Abstract Reporting System.

\section{Setting}

State of Washington, USA.

\section{Participants}

5595 case patients (infants readmitted to hospital during the first month of life) and 23439 control infants (no readmission during the first month of life and matched for year of birth) were identified from a cohort of 310578 births in Washington state between 1991 and 1994. Exclusion criteria were gestational age $<36$ weeks, multiple births, caesarean delivery, transfer to another hospital, or serious medical conditions including respiratory distress syndrome, pneumonia, sepsis, meconium aspiration, and some rare conditions such as extrophy of the bladder or double outlet right ventricle.

\section{Assessment of risk factors}

Length of hospital stay at birth was defined as early discharge (discharged within $30 \mathrm{~h}$ of birth) and later discharge (discharged 30-78 h after birth).

\author{
Main outcome measure \\ Hospital readmission at 7, 14, and 28 days after birth.
}

\section{Main results}

4971 newborn infants (17\%) were discharged early. Newborn infants who were discharged early were more likely than those who were discharged later to be readmitted to hospital at 7 days (odds ratio [OR] 1.28, 95\% CI 1.11 to 1.47 ), 14 days (OR 1.16, CI 1.03 to 1.32 ), and 28 days (OR 1.12 , CI 1.00 to 1.25 ) (adjusted for birth year, gestational age, maternal race/ ethnicity, insurance payer, maternal diabetes, premature rupture of membranes, pregnancy complications, sex, and parity). Analysis of a subgroup of healthy, liveborn singleton infants showed similar results for 7 days (OR 1.63, CI 1.35 to 1.96), 14 days (OR 1.34, CI 1.14 to 1.58), and 28 days (OR 1.22, CI 1.06 to 1.41) (adjusted for birth year, gestational age, maternal race/ ethnicity, insurance payer, pregnancy complications, sex, and parity).

\section{Conclusion}

Early discharge of newborn infants $<30$ hours after birth was associated with an increased risk of readmission to hospital at 7 , 14, and 28 days after birth.

Sources of funding: National Research Service Award; University of Washington, Seattle; and the Children's Research Endowment Fund, Children's Hospital and Medical Center, Seattle.

For article reprint:Dr L L Liu, Childhood Asthma Study Team, 146 N Canal, Suite 300, Seattle, WA 98103-8652, USA. Fax +1 2065435771. (commentary continued from page 41) and newborn babies before discharge. Screening criteria should be used to identify populations at increased risk and to assess readiness for discharge by considering biological, developmental, and psychosocial factors. Discharge decisions should not focus solely on timing but on readiness, which is individually determined.

Neither of the 2 studies gave information on services provided after discharge. Because early discharge was associated with increased risk of readmission for certain conditions, nurses need to provide close individual follow up within the first days of life. This would enable early identification of problems and subsequent intervention as well as promotion of child and family health and wellbeing. A range of comprehensive, flexible, and responsive postpartum discharge services which focus on neonatal and maternal assessment, breast feeding promotion, and teaching of infant care are needed. Given the current underfunding of community based postpartum discharge services, nurses must advocate for policy development and provision of funding for this range of options that ensures assessment and intervention at reasonable time intervals. Research will show whether the type, timing, intensity, and location of postpartum discharge follow up services are more crucial than the timing of hospital discharge.

Early postpartum discharge requires collaboration across sectors (hospitals, primary care, public health, and other community based programmes) and among providers (nurses, physicians, midwives, and other healthcare providers). Nurses can take a leadership role in collaborative planning and partnership building to create a "seamless" system of care and support for new mothers and their babies.

Nurses also have an important part to play in data collection and continuing research in this area. They can advocate for, and participate in, rigorous studies of sufficient size which examine the effect of different hospital stays and postdischarge practices on a range of outcomes for mothers and babies in diverse populations and settings. Only then can we inform practice, policy, and funding decisions.

Mary Lou Walker, RN, MHSc Manager, Public Health Nursing and Education Services, Toronto Public Health Toronto, Ontario, Canada

1 Grullon KE, Grimes DA. Obstet Gynecol $1997 ; 90: 860-5$

2 Braveman P, Egerter S, Marchi K, et al. Pediatrics 1995;96:716-26. 


\section{Promoting research utilisation in nursing: the role of the individual, organisation, and environment}

Evidence-based nursing integrates the best evidence from research with clinical expertise, patient preferences, and existing resources into decision making about the health care of individual patients. ${ }^{1}$ To practise evidence-based nursing, clinical nurses need effective strategies for extracting relevant information from the many publications that are currently available. The quality of information that nurses demand and how effectively they evaluate and use it for clinical decision making will influence patient outcomes and, ultimately, the part nurses play in the delivery of health care.

The term "information society" was introduced in the 1980s to describe the information explosion precipitated by new technologies. ${ }^{2}$ Nursing practice is information intensive. Even 10 years ago, Mowry and Korpman estimated that nurses spent $40 \%$ of their time on information related tasks. ${ }^{3}$ The rapid growth of nursing information means that nurses cannot rely on knowledge acquired as students and must constantly update their practice.

Computerisation is simultaneously a major cause of the information explosion and a means of managing it. Already, computer systems enable healthcare professionals to access patient databases, research databases, clinical guidelines, and care protocols. The internet has emerged as a formidable medium for information provision and exchange. The next challenge for the software industry is to devise an effective means of organising the available information so that discriminating users can access it effectively.

In the 1980s, when research was finally recognised as an integral part of professional nursing, concern developed about the gap between research and practice. Researchers began to investigate barriers to research utilisation, using a variety of individual and organisational variables to study diffusion of information throughout organisations. ${ }^{4}$ They also surveyed nurses about their attitudes, resources, and experiences related to research utilisation, ${ }^{6}$ and experimented with different approaches to improving research utilisation. ${ }^{78}$

\section{How do nurses currently use information?}

Studies of nurses' use of printed materials show differences in awareness of research and availability of information resources, both of which are often limited in small hospitals and primary care settings. ${ }^{9}{ }^{10}$ One study found that, compared with small hospitals ( $\leqslant 500$ beds), hospitals with $>500$ beds were more likely to have nursing research coordinators, access to nursing research experts and nursing faculty, nursing research committees, libraries with nursing research journals, and to implement research utilisation programmes for their staff nurses. ${ }^{9}$ Other surveys suggest that most nurses read clinical or technical journals rather than research journals ${ }^{11}$ and rarely visit libraries. ${ }^{12}$ Nurses engaged in education or research, however, use these resources as well as indexes and computerised databases such as CINAHL and Medline. ${ }^{13}$

Patient specific data and reference sources are increasingly available in computerised hospital informatics systems. Nurses are willing to use these resources for clinical decision making and patient teaching when the information is relevant and the system is user friendly. In one study, ${ }^{13}$ nurses used online information to:

- Answer questions related to nursing diagnoses

- Prepare care plans

- Learn about drugs and disease processes

- Obtain information for patients

- Validate knowledge

- Investigate new interests

- Promote professional development.

Drug databases and nursing texts were used most frequently. Novice users accessed online information during night shifts or quiet times. More expert users accessed information on all shifts, but used patient specific, quick references during the day. Little is known about nurses' use of the internet, including whether they use it to answer clinical questions at home, in practice, or both. At 1 site, staff accessed internet nursing sites during quiet periods on the night shift.

\section{Unharnessing the power of information for practice}

Nurses are generally positive about evidence-based practice, but we know little about what determines their use of research findings. We do know that individual research utilisation is facilitated by organisational commitment to using research evidence. A systematic review of the literature on research utilisation, ${ }^{14}$ found that organisational factors explained $80-90 \%$ of the variance in research utilisation, environmental factors accounted for $5-10 \%$ of the variance, and individual characteristics contributed only 1-3\%. Multifaceted interventions directed at the individual, the organisation, and the environment are most likely to lead to changes in practice. ${ }^{15}$

Numerous researchers have investigated the causes of the research-practice gap and proposed solutions. Based on a literature review, Funk et al identified barriers to research utilisation which included the individual nurse, the organisation, the quality of the research, and the way that research results are communicated. ${ }^{16}$ Surveys using Funk's Barrier Scale showed a widespread recognition of these barriers. ${ }^{17}$ Reports on programmes aimed at promoting research utilisation suggest possible strategies to overcome them. ${ }^{7}$

\section{The individual nurse}

NETWORKING

Nurses who participate in research related projects promote research utilisation and are more likely than other nurses to apply research in practice. ${ }^{18}$ Nurses who understand the importance of evidence-based nursing might act as mentors to colleagues who are unaware of its potential impact on practice or the range of information available. Once persuaded, nurses can collaborate to promote research utilisation in the workplace. Peer mentoring and coaching are effective strategies for assisting colleagues to search for, evaluate, and apply information. ${ }^{13}{ }^{19}$ Nurses with access to the internet can pass on information, and groups of nurses can form journal clubs to share reading on topics of clinical interest. 


\section{PROMOTING ACCESSIBILITY}

Because the accessibility and quality of information have implications for the efficacy of clinical decision making, it is important that workplace reference materials are current and user friendly and that protocols and guidelines are based on the best research evidence. Evaluating and updating personal collections is also important.

\section{MANAGING TIME}

Prioritisation is important when time is limited. Strategic choices may include reading a research article or critical review instead of a clinical journal, or taking a course on how to understand or apply research findings. A trip to a health sciences or medical library to learn how to use CINAHL, Medline, or the Cochrane Library will make it easier to find useful articles. Pooling skills and resources can also increase research utilisation.

\section{The organisation}

Organisational characteristics, including size, location, and centralisation of decision making, have been associated with research utilisation, but conclusions about the importance of specific variables are inconsistent, ${ }^{14}$ perhaps because their effects vary in different settings. Programmes intended to improve research utilisation have been most successful when administrative support was available. ${ }^{1319}$ Evidence-based practice has been realised most completely in institutions that have adopted it as policy and have integrated it at all organisational levels. ${ }^{20}{ }^{21}$ At the University of Iowa Hospitals, an infrastructure supporting research use encompassed both high level management and frontline nurses. Duties relating to research utilisation were included in job descriptions; evidence-based practice was linked to quality assurance; and appropriate education was provided. Staff who used research to solve clinical problems were recognised and rewarded. The Iowa model used triggers to alert nurses to clinical problems in their workplaces and specified processes for solving them. Clinical nurses were allocated time and resources to be involved in all aspects of research utilisation including problem identification, assessment of evidence, change planning, implementation, and evaluation of change. Because the organisation placed a high priority on research utilisation, nurses were motivated to become involved. ${ }^{21}$

\section{The environment}

An information friendly environment links the clinical nurse to a variety of information brokers, the healthcare literature, and other evidence-based resources. The internet provides potential global connectedness for all nurses.

The development of tools for evidence-based nursing requires time and resources and is dependent on collaboration among healthcare organisations and academic institutions. As communication throughout the global healthcare community improves, pooling expertise on national and international levels becomes increasingly feasible. Centres for evidence-based nursing have been established in a number of countries and plans are underway to create an international centre with links to centres worldwide. These centres will provide access to critically appraised materials for nurses and organisations. Health science libraries are important resources for clinicians. Besides keeping up with new information resources, the librarian's role includes helping users to master information handling skills, and using technology to organise, synthesise, and filter information for scholarly, clinical, and institutional decision making.

\section{Conclusions}

In the future, information systems will integrate clinical data about patient treatments and preferences, available resources, expert opinion, and research evidence to provide nurses with current, patient specific information to address complex clinical issues. Nurses will be able to plan treatment with knowledgeable patients who will also have access to health related resources. Improved organisational and environmental support will be critical for nurses to work with increasingly sophisticated information systems.

JOAN ROYLE, RN, MScN

School of Nursing, JENNIFER BLYTHE, PhD, MLS

Faculty of Health Sciences,

McMaster University,

Hamilton, Ontario, Canada

1 DiCenso A, Cullum N, Ciliska D. Implementing evidence-based nursing: some misconceptions [implementation forum]. Evidence-Based Nursing 1998 Apr;1:38-40.

2 Naisbitt J. Megatrends: ten new directions for transforming our lives. New York: Warner Books, 1984

Mowry MM, Korpman RA. Evaluating automated information systems. Nurs Econ 1987;5:7-12.

4 Brett JL. Organizational integrative mechanisms and adoption of innovations by nurses. Nurs Res 1989;38:105-10.

5 Champion VL, Leach A. Variables related to research utilization in nursing: an empirical investigation. I Adv Nurs 1989;14:705-10.

6 Funk SG. Champagne MT, Weise RA, et al. Barriers to using research findFunk SG, Champagne MT, Weise RA, et al. Barriers to using research find-
ings in practice: the clinician's perspective. Appl Nurs Res 1991;4:90-5.

ings in practice: the clinician's perspective. Appl Nurs Res 1991;4:90-5.
Goode CJ, Lovett MK, Haves JE, et al. Use of research-based knowledge in Goode CJ, Lovett MK, Hayes JE, et al. Use of
clinical practice. J Nurs Adm 1987;17:11-18.

clinical practice. J Nurs Adm 1987;17:11-18. 8 Royle JA, Blythe J, Potvin C, et al. Litera

9 Mitchell A, Janzen K, Pask E, et al. Assessment of nursing research utilization needs in Ontario health agencies. Can J Nurs Adm 1995;8:77-91.

10 Royle JA, Blythe J, DiCenso A, et al. Do nurses have the information resources and skills for research utilization? Can J Nurs Adm 1997;10:9-30.

11 Blythe J, Royle JA. Assessing nurses' information needs in the work environment. Bull Med Libr Assoc 1993;81:433-5.

12 Stephens LC, Selig CL, Jones LC, et al. Research application: teaching staff nurses to use library search strategies. J Contin Educ Nurs 1992:23:24-8.

13 Royle JA, Blythe J, Boblin-Cummings S, et al. Nursing for the twenty-first century: using information technology to enhance nursing practice. Hamilton,
Ontario: McMaster University, 1997 .

14 Dobbins M, Ciliska D, DiCenso A. Dissemination and use of research evidence for policy and practice: a framework for developing, implementing, and evaluating strategies. A report prepared for the Dissemination and Utilization Model Advisory Committee of the Canadian Nurses Association and Health Canada, 1998

15 Thomson MA. Closing the gap between nursing research and practice [implementation forum]. Evidence-Based Nursing 1998 Jan; 1:7-8.

16 Funk SG, Champagne MT, Wiese R, et al. BARRIERS: the barriers to utilization scale. Appl Nurs Res 1991;4:39-45.

17 Funk SG, Tornquist EM, Champagne MT. Barriers and facilitators of research utilization. An integrative review. Nurs Clin North Am 1995;30: 395-407.

18 Bostrom J, Suter WN. Research utilization: making the link to practice. $J$ Nurs Staff Dev 1993;9:28-34.

19 Logan J, Davies B. The staff nurse as research facilitator. Can J Nurs Adm 1995;8:92-110.

20 MacGuire JM. Putting nursing research findings into practice: research utilization as an aspect of the management of change. $J$ Adv Nurs

21 Titler MG, Kleiber C, Steelman V, et al. Infusing research into practice to promote quality care. Nurs Res 1994;43:307-13.

Erratum: In the April 1998 issue of Evidence-Based Nursing, the 2 abstracts related to early hospital discharge (Edmonson et al and Liu $e t a l)$ appeared in the treatment section, but they should have appeared in the causation section. 